\title{
Extensive Distortion and Dysfunction of an Edwards Valeo Lifestent Placed for Stenosis of the Superior Vena Cava
}

\author{
Ranjit Aiyagari, ${ }^{*}$ MD, FACC, FSCAI \\ We present a case of multiple interventions to treat symptomatic stenosis of the supe- \\ rior vena cava in a child, with end-stage renal disease and a history of long-term \\ hemodialysis, who had required indwelling central venous catheters for several years. \\ The stenosis was successfully treated with an Edwards Valeo Lifestent, but recurrent \\ symptomatic superior vena caval stenosis developed within 5 months, requiring repeat \\ catheterization and stent placement. @ 2011 Wiley-Liss, Inc.
}

Key words: pediatric interventions; restenosis; stent; SVC syndrome

\section{INTRODUCTION}

Stenosis of the superior vena cava (SVC) is an unusual condition in pediatric patients. When severe, it can lead to symptomatic SVC syndrome, which includes swelling of the face, neck, and arms, and it can potentially lead to life-threatening dyspnea and dysphagia [1]. The most common risk factors for the development of SVC stenosis in pediatric patients without congenital heart disease include thoracic neoplasms (both malignant and benign) and central venous catheterization with associated scarring or thrombosis [2].

Endovascular stent therapy for SVC syndrome was first described by Charnsangavej [3] and has become a standard approach for therapy over the last two decades. In a recent series of patients with SVC syndrome and malignancy treated with a variety of different stents, the technical success rate for stent placement was $100 \%$, with a procedural complication rate of $18 \%$, and a 1 -year restenosis rate of $12 \%$ [4].

We present a case of multiple interventions in a 9year-old child with end-stage renal disease who developed catheter-associated thrombosis and symptomatic, high-grade SVC stenosis. Treatment with a Valeo Lifestent (Edwards Lifesciences, Irvine, CA) resulted in a suboptimal medium-term outcome and required semiurgent reintervention.

\section{CASE REPORT}

The patient was born prematurely at 32 weeks gestation with congenital renal dysplasia and chronic polyuric renal insufficiency. Peritoneal dialysis was instituted at age 8 months, but this approach was aban- doned due to repeated episodes of peritonitis. To institute hemodialysis, a 4.2-French Broviac catheter (Bard, Salt Lake City, UT) was placed in the right subclavian vein at age 20 months. This catheter malfunctioned and was removed 11 days after placement. An 8-French PermCath catheter (Covidien, Mansfield, MA) was placed in the right internal jugular vein at age 29 months. Over the ensuing 10 months, the patient developed multiple episodes of catheter malfunction, and the right internal jugular venous catheters were repeatedly replaced over a wire. When the patient was 39 months old, the right internal jugular vein was found to be occluded with drainage via collaterals. The patient thereafter had multiple venous catheters placed and replaced in the right subclavian vein. The patient then underwent cadaveric renal transplantation at age 44 months.

Approximately 2 years after renal transplant, at age 5 , the patient developed focal segmental glomerulosclerosis. He was treated medically over the following 2

\section{Division of Pediatric Cardiology, University of Michigan, Ann} Arbor, Michigan

Conflict of interest: Nothing to report.

*Correspondence to: Ranjit Aiyagari, MD, FACC, FSCAI, Division of Pediatric Cardiology, University of Michigan, L1242 Women's Hospital/SPC 5204, 1500 E. Medical Center Dr., Ann Arbor, MI 48109. E-mail: ranjita@umich.edu

Received 18 January 2011; Revision accepted 19 February 2011

DOI 10.1002/ccd.23088

Published online 22 July 2011 in Wiley Online Library (wileyonlinelibrary.com) 


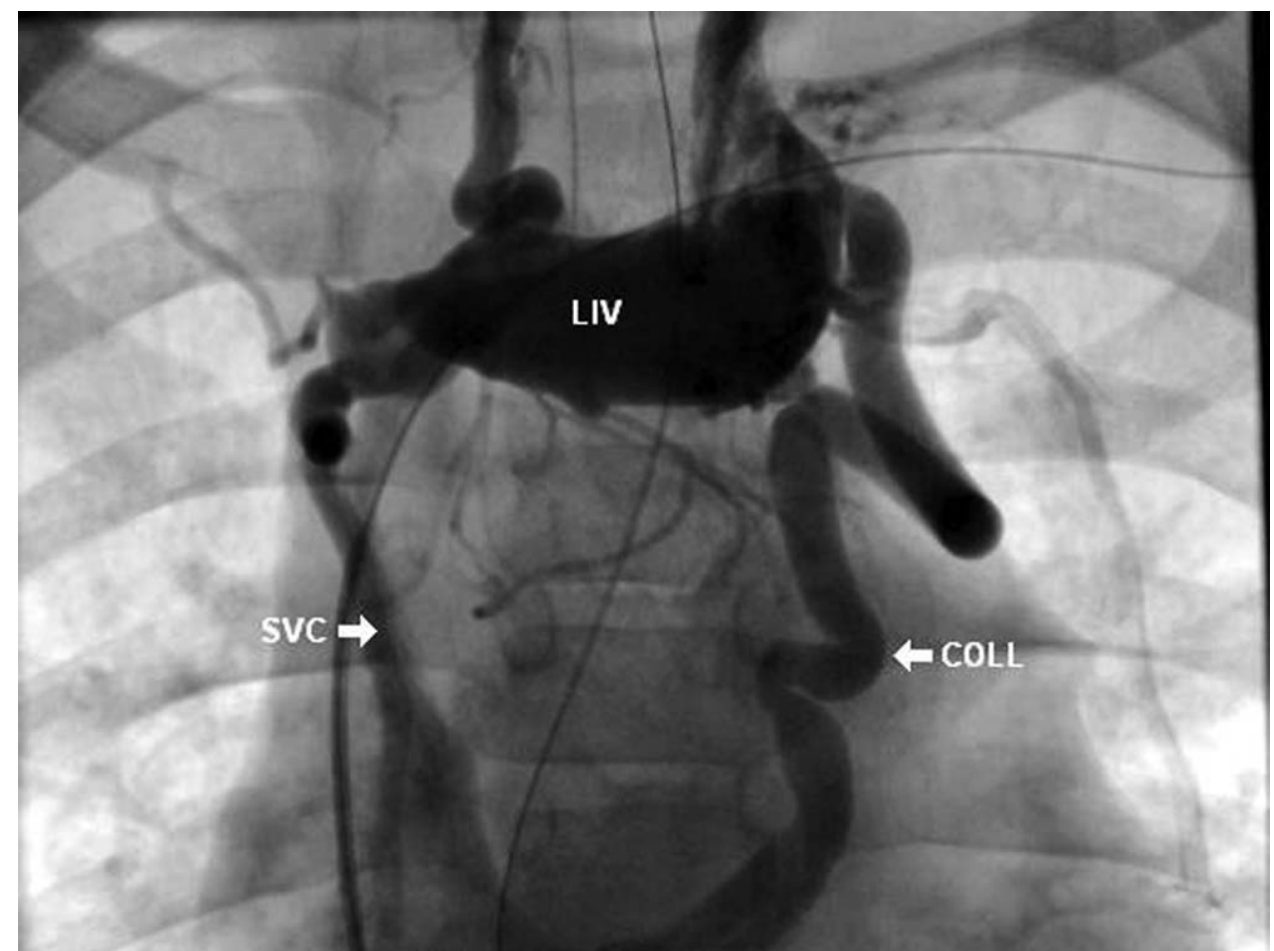

RA

Fig. 1. Posteroanterior angiographic image showing diffuse, long-segment narrowing of the superior vena cava (SVC) along its entire length. There is a dilated left innominate vein (LIV). There is a large collateral (COLL) decompressing the LIV. The right atrium (RA) is seen below the SVC.

years, but due to a need to resume hemodialysis, he had a tunneled catheter placed in the left internal jugular vein at age 7 . This became infected and was removed 5 months later, at which time an attempt was made to reaccess the right internal jugular vein, which was again found to be occluded. The patient underwent construction of a left upper extremity brachiobasilic arteriovenous fistula for continued hemodialysis.

At age 9, the patient was noted to have mild signs of SVC syndrome including facial edema. An echocardiogram showed probable thrombus within the SVC with an estimated mean gradient of $20 \mathrm{~mm} \mathrm{Hg}$. There was a small linear thrombus in the left innominate vein without obstruction. A magnetic resonance venogram was performed and showed long-segment SVC stenosis for a length of $2.7 \mathrm{~cm}$ with a minimum diameter of 2$3 \mathrm{~mm}$; however, image quality was limited due to sus- ceptibility artifact from a foreign body in the right lower neck. A chest radiograph showed this to be a 2.5-cm-long fragment of a J-tipped wire near the area of the mid right internal jugular vein.

Because of the new symptoms, the patient was taken to the cardiac catheterization laboratory at age 9 years 6 months. Under general anesthesia, an 8-French sheath was placed in the right femoral vein. Occlusion of the right internal jugular vein was documented near the site of the retained wire fragment. A 6-French sheath was placed in the left internal jugular vein. The mean pressure in the left innominate vein was $24 \mathrm{~mm}$ $\mathrm{Hg}$, while the right atrial mean pressure was $6 \mathrm{~mm} \mathrm{Hg}$. Venography showed a complex, severe SVC stenosis with multiple large venous collaterals draining the upper body (Fig. 1). The minimum diameter of the SVC was $3.0 \mathrm{~mm}$ and the stenotic segment was $3.0 \mathrm{~cm}$ in length. 
Balloon angioplasty was initially undertaken, with multiple inflations of an $8 \mathrm{~mm} \times 3 \mathrm{~cm} \mathrm{Z-Med} \mathrm{II} \mathrm{bal-}$ loon (NuMed, Hopkinton, NY) to pressures of $10 \mathrm{~atm}$, resulting in a residual waist and no improvement in stenosis. Therefore, the decision was made to place a stent. A Valeo (Edwards Lifesciences, Irvine, CA) $10 \mathrm{~mm} \times$ $36 \mathrm{~mm}$ premounted stent was chosen because of the convenience of a premounted stent, the previously reported high radial strength of this stent, and its ability to be dilated in the future to $20 \mathrm{~mm}$ [5]. The stent was deployed across the area of stenosis with balloon inflation to 6 atm. Because there was some residual narrowing of the upper part of the stent, the stent was re-expanded with a single inflation to 12 atm of a $10 \mathrm{~mm} \times 3 \mathrm{~cm}$ Z-med II balloon (NuMed, Hopkinton, NY), with no apparent waist on the balloon at maximal inflation. Fluoroscopy showed mild residual stenosis in the upper third of the stent with no obvious stent fracture (Fig. 2), and angiography showed good flow through the stent with no significant residual flow through venous collaterals. Pressure measurement showed a residual gradient of $4 \mathrm{~mm} \mathrm{Hg}$. The procedure was concluded, and the patient was maintained on Lovenox anticoagulation and followed clinically as an outpatient.

In the first few months following stent placement, the patient's facial swelling markedly decreased. An echocardiogram 5 weeks after stent placement showed an estimated residual mean gradient of $4.5 \mathrm{~mm} \mathrm{Hg}$. However, 5 months after stent placement, he again began to have periorbital edema. Repeat echocardiogram at that time showed that the mean estimated pressure gradient across the SVC was again $17-21 \mathrm{~mm} \mathrm{Hg}$.

The patient, now age 9 years 11 months, was again brought to the cardiac catheterization laboratory. Under general anesthesia, access was again obtained in the same locations with the same size sheaths, and the left innominate vein mean pressure was measured at 23 $\mathrm{mm} \mathrm{Hg}$ with a right atrial mean pressure of $7 \mathrm{~mm} \mathrm{Hg}$. Fluoroscopy showed extensive stent distortion and disruption (Fig. 3A) and angiography showed recurrent severe SVC stenosis with significant in-stent restenosis (Fig. 3B). A $10 \mathrm{~mm} \times 36 \mathrm{~mm}$ iCAST stent (Atrium Medical, Hudson, NH) was then placed over a 0.035in. Amplatz Super Stiff wire (Boston Scientific, Watertown, MA) into the area of the existing fractured stent. This was fully expanded with 8 atm of balloon pressure. The new stent was then re-expanded with a 12 $\mathrm{mm} \times 4 \mathrm{~cm}$ Z-Med II balloon (NuMed, Hopkinton, NY) inflated to $10 \mathrm{~atm}$. There was no waist with this inflation. Angiography showed significant improvement (Fig. 4), and repeat pressure measurements showed a residual gradient of $3 \mathrm{~mm} \mathrm{Hg}$.

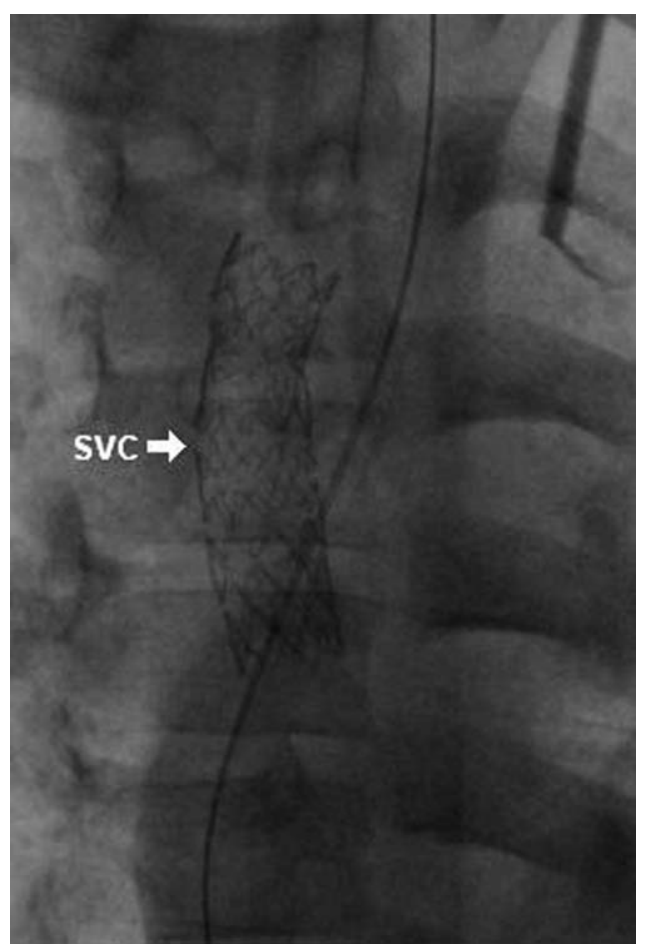

Fig. 2. Posteroanterior fluoroscopic image showing a $10 \mathrm{~mm}$ $\times 36 \mathrm{~mm}$ Edwards Valeo stent within the superior vena cava (SVC) after implantation. The stent appears intact with mild narrowing along its superior $1 / 3$.

The patient is free of symptoms of SVC syndrome 6 months after placement of the iCAST stent. An echocardiogram performed 2 months after the final procedure showed a residual estimated mean gradient of 5 $\mathrm{mm} \mathrm{Hg}$.

\section{DISCUSSION}

This patient is representative of many children who are chronically dependent on central venous access, whether for dialysis, medication administration, or other purposes. Because of his high activity level, potential for being less careful, and growth, the child required multiple line exchanges and replacements. Combined with the retained wire fragment and associated scar formation, this resulted in a clinically important stenosis of the SVC.

SVC stenosis represents an ideal case for intervention with intravascular stent placement. The relatively straight course of the vessel and the ease of obtaining access from above, below, or both facilitate recanalization in the setting of total occlusion, as well as stent placement with concomitant angiography.

The Valeo Lifestent is a premounted and open-cell stainless steel triple helical stent that comes in diameters ranging from 6 to $10 \mathrm{~mm}$ with lengths ranging from 18 to $56 \mathrm{~mm}$. The larger $(9-10 \mathrm{~mm})$ diameter stents can be 

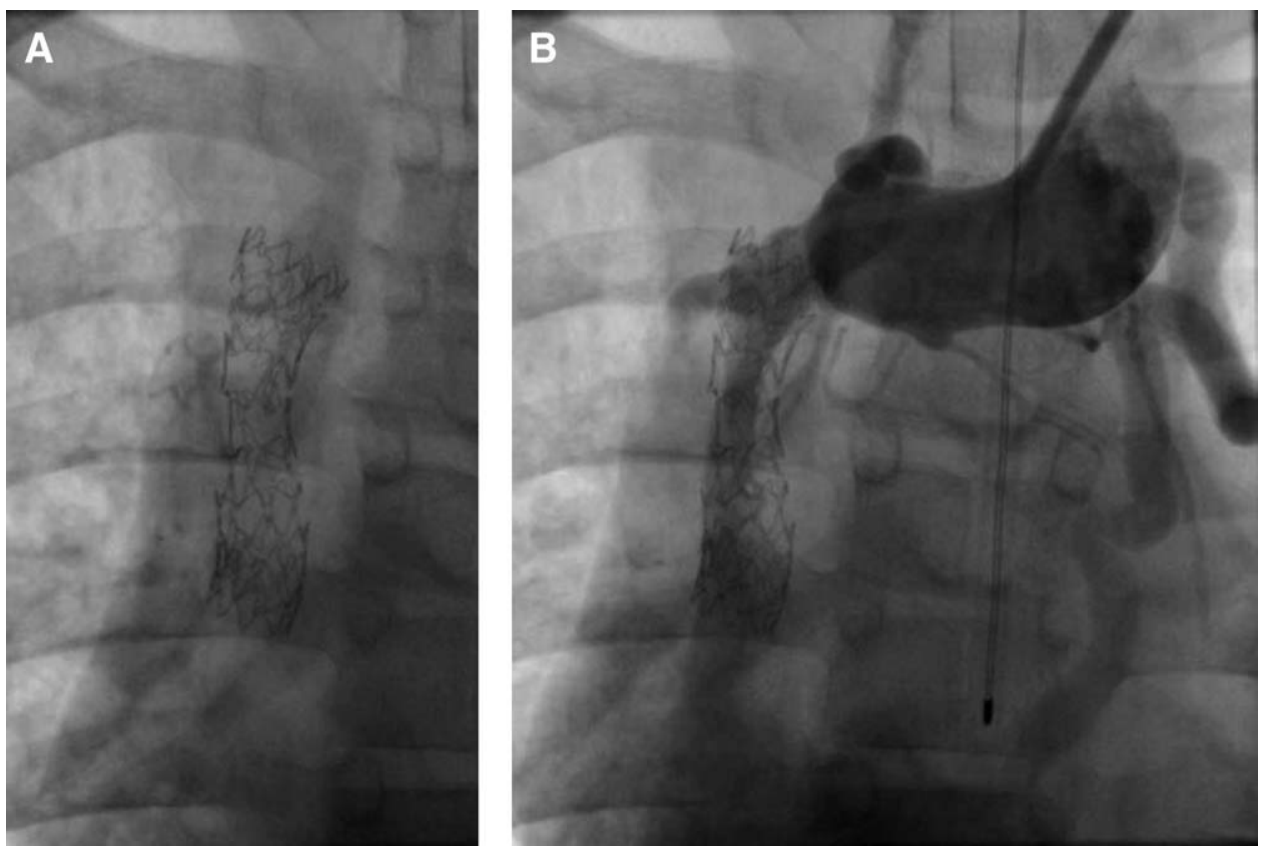

Fig. 3. (A) Posteroanterior fluoroscopic image showing the $10 \mathrm{~mm} \times 36 \mathrm{~mm}$ Edwards Valeo stent within the superior vena cava 5 months after implantation. There is severe distortion and deformation of the stent along its entire length. (B) Posteroanterior angiographic image showing the distorted stent with recurrent, severe stenosis despite appropriate stent location. There is also significant in-stent stenosis.

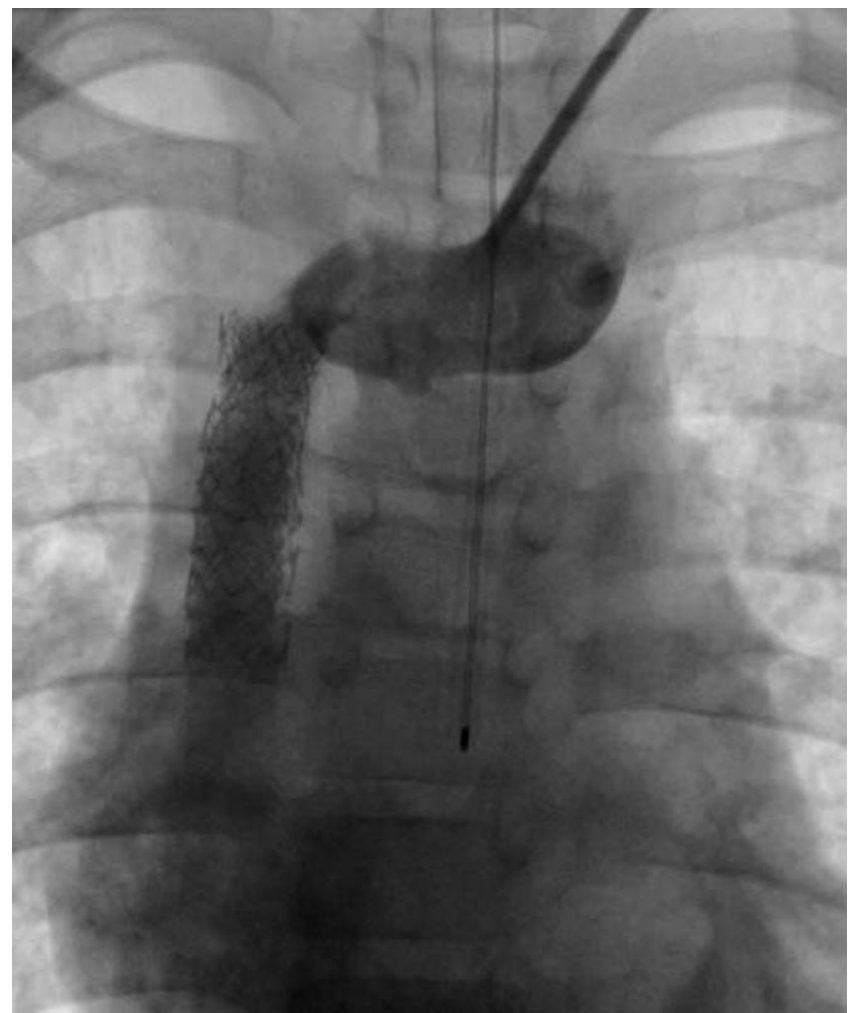

Fig. 4. Posteroanterior angiographic image showing good patency of the superior vena cava after placement of a $10 \mathrm{~mm}$ $\times 36 \mathrm{~mm}$ iCAST stent within the original stent and redilation to $12 \mathrm{~mm}$. delivered through 7-French sheaths, and overexpansion to $18 \mathrm{~mm}$ diameters results not in foreshortening but instead in larger gaps between the open cells [5].

This case illustrates that in the setting of vascular stenosis with significant scar formation, the Valeo Lifestent, even when not expanded above its nominal diameter, may lack sufficient radial strength to achieve lasting relief. This patient would likely have achieved longer lasting relief of stenosis with use of a stent with higher radial strength. This important limitation of the Valeo Lifestent should be kept in mind.

\section{REFERENCES}

1. Kishi K, Sonomura T, Mitsuzane K, Nishida N, Yang RJ, Sato M, Yamada R, Shirai S, Kobayashi H. Self-expandable metallic stent therapy for superior vena cava syndrome: Clinical observations. Radiology 1993;189:531-535.

2. Solomon N, Wholey MH, Jarmolowski CR. Intravascular stents in the management of superior vena cava syndrome. Cathet Cardiovasc Diagn 1991;23:245-252.

3. Charnsangavej C, Carrasco CH, Wallace S, Wright KC, Ogawa K, Richli W, Gianturco C. Stenosis of the vena cava: Preliminary assessment of treatment with expandable metallic stents. Radiology 1986;161:295-298.

4. Cho TH, Janho K, Mohan IV. The role of stenting the superior vena cava syndrome in patients with malignant disease. Angiology 2011;62:248-252.

5. Stern H, Baird C. A premounted stent that can be implanted in infants and re-dilated to $20 \mathrm{~mm}$ : Introducing the Edwards Valeo Lifestent. Cathet Cardiovasc Diagn 2009;74:905-912. 